\title{
The Escitalopram versus Electric Current Therapy for Treating Depression Clinical Study (ELECT-TDCS): rationale and study design of a non-inferiority, triple-arm, placebo-controlled clinical trial
}

\author{
Estudo clínico Escitalopram versus Eletroterapia no Tratamento da Depressão \\ (ELECT-TDCS): racional e desenho de estudo de um ensaio de não inferioridade, \\ de três braços, placebo-controlado
}

André Russowsky Brunoni', Bernardo Sampaio-Junior", Adriano Henrique Moffa"', Lucas Borrione', Barbara Schwair Nogueira'I', Luana Vanessa Marotti Aparíciov, Beatriz Veronezi ${ }^{\mathrm{V}}$, Marina Moreno "', Raquel Albano Fernandes ${ }^{\mathrm{VI}}$, Diego Tavares ${ }^{\mathrm{VII}}$, Priscila Vilela Silveira Bueno ${ }^{\mathrm{VII}}$, Ole Seibt ${ }^{\mathrm{VIII}}$, Marom Bikson ${ }^{\prime \mathrm{x}}$, Renerio Fraguas' ${ }^{\mathrm{x}}$, Isabela Martins Benseñor ${ }^{\mathrm{xI}}$

Faculdade de Medicina da Universidade de São Paulo (FMUSP), São Paulo, Brazil

'MD, PhD. Attending Physician, Interdisciplinary Neuromodulation Service, Interdisciplinary Center for Applied Neuromodulation, Hospital Universitário (HU), and Service of Interdisciplinary Neuromodulation, Laboratory of Neurosciences (LIM-27), Department and Institute of Psychiatry, Universidade de São Paulo (USP), São Paulo, Brazil. "MD. Interdisciplinary Neuromodulation Service, Interdisciplinary Center for Applied Neuromodulation, $\mathrm{HU}$, and Service of Interdisciplinary Neuromodulation, Laboratory of Neurosciences (LIM-27), Department and Institute of Psychiatry, USP, São Paulo, Brazil.

"'BA, MSc. Interdisciplinary Center for Applied Neuromodulation, HU, USP, São Paulo, Brazil. "MD, MSc. Interdisciplinary Center for Applied Neuromodulation, HU and Department and Institute of Psychiatry, USP, São Paulo, Brazil.

vMD, MSc. Interdisciplinary Center for Applied Neuromodulation, HU, USP, São Paulo, Brazil. uPsychology Student, Interdisciplinary Center for Applied Neuromodulation, HU, USP, São Paulo, Brazil.

"MD. Medical Resident and Student, Interdisciplinary Neuromodulation Service, Interdisciplinary Center for Applied Neuromodulation, HU and Department and Institute of Psychiatry, USP, São Paulo, Brazil. "nIMSc. Researcher, Department of Biomedical Engineering, City College of City University of New York, New York, USA.

xphD. Head, Department of Biomedical Engineering, City College of City University of New York, New York, USA. ${ }^{x} \mathrm{MD}, \mathrm{PhD}$. Assistant Professor, HU and Department and Institute of Psychiatry, USP, São Paulo, Brazil. ${ }^{\times M D}$, PhD. Assistant Professor, HU, USP, São Paulo, Brazil.

\section{KEY WORDS:}

Depressive disorder, major.

Electric stimulation therapy.

Citalopram.

Randomized controlled trial.

Biological markers.

\section{PALAVRAS-CHAVE:}

Transtorno depressivo maior.

Terapia por estimulação elétrica.

Citalopram

Ensaio clínico controlado aleatório.

Marcadores biológicos.

\section{ABSTRACT}

CONTEXT AND OBJECTIVE: Major depressive disorder (MDD) is a common psychiatric condition, mostly treated with antidepressant drugs, which are limited due to refractoriness and adverse effects. We describe the study rationale and design of ELECT-TDCS (Escitalopram versus Electric Current Therapy for Treating Depression Clinical Study), which is investigating a non-pharmacological treatment known as transcranial direct current stimulation (tDCS). DESIGN AND SETTING: Phase-III, randomized, non-inferiority, triple-arm, placebo-controlled study, ongoing in São Paulo, Brazil.

METHODS: ELECT-TDCS compares the efficacy of active tDCS/placebo pill, sham tDCS/escitalopram $20 \mathrm{mg} /$ day and sham tDCS/placebo pill, for ten weeks, randomizing 240 patients in a 3:3:2 ratio, respectively. Our primary aim is to show that tDCS is not inferior to escitalopram with a non-inferiority margin of at least $50 \%$ of the escitalopram effect, in relation to placebo. As secondary aims, we investigate several biomarkers such as genetic polymorphisms, neurotrophin serum markers, motor cortical excitability, heart rate variability and neuroimaging. RESULTS: Proving that tDCS is similarly effective to antidepressants would have a tremendous impact on clinical psychiatry, since tDCS is virtually devoid of adverse effects. Its ease of use, portability and low price are further compelling characteristics for its use in primary and secondary healthcare. Multimodal investigation of biomarkers will also contribute towards understanding the antidepressant mechanisms of action of tDCS.

CONCLUSION: Our results have the potential to introduce a novel technique to the therapeutic arsenal of treatments for depression.

CLINICAL TRIAL REGISTRATION: ClinicalTrials.Gov NCT01894815.

\section{RESUMO}

CONTEXTO E OBJETIVO: O transtorno depressivo maior (TDM) é uma condição psiquiátrica comum, tratada com medicamentos antidepressivos, os quais são limitados devido à refratariedade e efeitos adversos. Descrevemos o racional e o desenho do Estudo Clínico Escitalopram versus Eletroterapia no Tratamento da Depressão (ELECT-TDCS), que investiga um tratamento não farmacológico, conhecido como estimulação transcraniana por corrente contínua (ETCC). DESENHO E LOCAL: Ensaio de fase III, randomizado, de não inferioridade, de três braços, placebo-controlado, em execução em São Paulo, Brasil.

MÉTODOS: O estudo compara a eficácia da ETCC ativa/pílula placebo, ETCC simulada/escitalopram $20 \mathrm{mg} / \mathrm{dia}$ e ETCC simulada/pílula placebo durante 10 semanas, randomizando 240 pacientes em uma proporção 3:3:2, respectivamente. O objetivo principal é demostrar que a ETCC não é inferior ao escitalopram com uma margem de não inferioridade de pelo menos $50 \%$ do efeito de escitalopram em relação ao placebo. Como objetivos secundários, investigamos biomarcadores como polimorfismos genéticos, marcadores séricos, excitabilidade cortical motora, variabilidade da frequência cardíaca e neuroimagem.

RESULTADOS: Provar que ETCC é igualmente eficaz a antidepressivos teria um tremendo impacto na psiquiatria clínica, uma vez que a ETCC é praticamente isenta de efeitos adversos. Sua facilidade de uso, portabilidade e preço baixo são outras características atraentes para uso na atenção primária e secundária de saúde. A investigação multimodal de biomarcadores também contribuirá para a compreensão dos mecanismos de ação antidepressivos da ETCC. CONCLUSÃO: Os nossos resultados podem introduzir uma nova técnica no arsenal terapêutico do tratamento da depressão. REGISTRO DE ENSAIO CLÍNICO: ClinicalTrials.Gov NCT01894815. 


\section{INTRODUCTION}

Major depressive disorder (MDD) is a psychiatric condition with high prevalence and recurrence worldwide, ${ }^{1}$ and it is projected to be the second greatest cause of disability worldwide in 2020. ${ }^{2}$ However, antidepressant drugs are only moderately effective for MDD treatment. ${ }^{3}$ Moreover, common adverse effects can lead to treatment discontinuation and recrudescence of symptoms. ${ }^{4}$ For these reasons, novel treatment strategies are continuously pursued.

In this context, non-invasive brain stimulation therapies (electroceuticals) have been increasingly investigated as nonpharmacological MDD treatments, such as repetitive transcranial magnetic stimulation (rTMS), a technique that discharges potent electromagnetic fields through a coil placed over the patient's head. ${ }^{5}$ Repetitive TMS is an effective treatment for $\mathrm{MDD},{ }^{6,7}$ although issues such as high cost of application, discomfort and specialized technology limit widespread adoption. ${ }^{8}$

Transcranial direct current stimulation (tDCS) is a neuromodulatory technique that presents low cost, high portability and a benign profile of adverse effects and is relatively simple to use. ${ }^{9,10}$ It consists of applying a low-intensity electric current across the patient's head using two electrodes on the scalp. In a seminal study in 2000, Nitsche and Paulus demonstrated that anodal and cathodal tDCS applied over the motor cortex respectively increased and decreased motor cortical excitability, as measured by means of TMS motor-evoked potentials, ${ }^{11}$ thereby showing that $\mathrm{tDCS}$ could alter cortical excitability. ${ }^{12}$ Its effects depend not only on the polarity of the current, but also on other factors, such as baseline cortical activity, neuronal orientation and delivered current intensity and duration. ${ }^{13-15}$

The use of tDCS for MDD is based on findings from neuroimaging studies (such as Mayberg et al. ${ }^{16}$ ), which suggest that the depressive state is associated with hypoactivity of prefrontal areas, particularly the left dorsolateral prefrontal cortex (DLPFC), and that it can be resolved through an increase in the activity of this area. Therefore, treatment strategies that increase left DLPFC activity might treat depressive symptoms. This approach has been adopted and validated in studies using high-frequency (excitatory) rTMS $^{6}$ and anodal tDCS over the DLPFC. ${ }^{17}$ Alternatively, some trials have applied the cathode over the right DLPFC (such as Ferrucci et al. ${ }^{18}$ ), or have used low-frequency (inhibitory) rTMS over this region, ${ }^{19}$ in accordance with the prefrontal asymmetry theory of depression that states that the right DLPFC presents abnormally high activity during depressive episodes. ${ }^{20}$

Several non-controlled and controlled studies using tDCS for MDD have been published, from 2006 onwards. ${ }^{17}$ In the first randomized clinical trial (RCT) in this field, Fregni et al. ${ }^{21}$ showed the efficacy of active tDCS versus sham for ameliorating depressive symptoms, in a pilot study on 10 patients. More recent RCTs enrolling larger samples ${ }^{22,23}$ have shown that active tDCS was an effective treatment for MDD. Its efficacy was corroborated by a recent meta-analysis. ${ }^{24}$ However, exhaustive RCTs addressing tDCS efficacy are still needed, since some results from RCTs have been non-significant, ${ }^{25-27}$ the total number of subjects investigated is still low and the optimal parameter protocols for tDCS in MDD remain to be determined.

In a recent factorial, placebo-controlled trial named SELECT-TDCS (Sertraline versus Electric Current Therapy for Treating Depression Clinical Study), we assessed the efficacy of tDCS combined and compared with sertraline $50 \mathrm{mg} /$ day for treating 120 depressed patients. ${ }^{28}$ After two weeks of treatment and at the endpoint, the combined treatment was statistically superior to the other groups in terms of depression improvement and response and remission rates. Transcranial DCS was also superior to the other groups at the endpoint and not statistically different from sertraline ${ }^{23}$ (Figure 1). The main findings from this study were the synergistic effects of the combination of tDCS and sertraline and the efficacy of tDCS as monotherapy. However, although sertraline and tDCS efficacy did not statistically differ, we could not extend these results to claim similar efficacy for tDCS and antidepressant pharmacotherapy, because of study limitations such as the low dose of sertraline used, the relatively short trial duration, the finding that the sertraline group was not superior to placebo and the relatively underpowered comparison of tDCS versus sertraline.

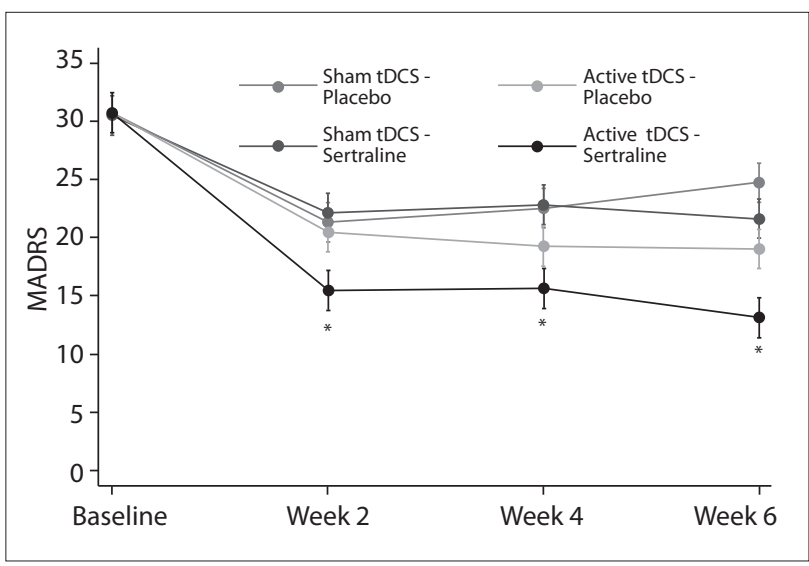

Figure 1. Main results of the SELECT-TDCS trial. The figure shows the primary outcome of the Sertraline versus Electric Current Therapy for Treating Depression Clinical Study (SELECT-TDCS). ${ }^{23}$ The $x$-axis represents depression scores measured using the Montgomery-Asberg depression rating scale (MADRS). The $y$-axis shows changes in depression scores over time according to the treatment group. Adapted from Brunoni et al. ${ }^{23}$ 


\section{OBJECTIVE}

Therefore, whereas we showed in SELECT-TDCS that tDCS combined with pharmacotherapy could enhance improvement of depression, the main study aim of ELECT-TDCS (Escitalopram versus Electric Current Therapy for Treating Depression Clinical Study) is to compare the efficacy of tDCS with a fully dosed, firstline antidepressant treatment (escitalopram $20 \mathrm{mg} /$ day).

In this paper, we describe the rationale, design and methodology of the ongoing ELECT-TDCS trial. The trial started in October 2013 and plans to enroll 240 patients by January 2017. As of November 2014, approximately 130 patients had already been recruited.

\section{METHODS}

\section{Overview}

The ELECT-TDCS trial randomizes patients into: sham-tDCS/ placebo-pill (placebo group), sham-tDCS/escitalopram (escitalopram group) and active-tDCS/placebo-pill (tDCS group). It was approved by the Local and National Ethics Committee (CAAE:10173712.3.0000.0076) of the University Hospital and Clinics Hospital of the University of São Paulo and is registered in clinicaltrials.gov (NCT01894815). All study participants provide written, informed consent for participation in the study.

Our null hypothesis is that the improvement, i.e. the difference in efficacy between baseline and endpoint measurements of the tDCS group $\left(\mu_{\mathrm{tDCS}}\right)$ will be less than or equal to $50 \%$ (retention fraction $f \leq 50 \%$ ) of the difference in the improvement between escitalopram and placebo $\left(\mu_{\text {drug }}-\mu_{\text {placebo }}\right)$. The study's primary aim is to prove the alternative hypothesis, i.e. that this difference is greater than $50 \%(f>50 \%)$.

$$
\begin{aligned}
& \mathrm{H}_{\mathrm{o}}: \mu_{\mathrm{tDCS}}-\mu_{\text {placebo }} \leq 50 \%\left(\mu_{\text {drug }}-\mu_{\text {placebo }}\right) \text { or } \\
& \mathrm{H}_{\mathrm{o}}: \mu_{\mathrm{tDCS}}-0.5 \mu_{\text {placebo }}-0.5 \mu_{\text {drug }} \leq 0 \\
& \mathrm{H}_{\mathrm{A}}: \mu_{\mathrm{tDCS}}-\mu_{\text {placebo }}>50 \%\left(\mu_{\text {drug }}-\mu_{\text {placebo }}\right) \text { or } \\
& \mathrm{H}_{\mathrm{A}}: \mu_{\mathrm{tDCS}}-0.5 \mu_{\text {placebo }}-0.5 \mu_{\text {drug }}>0
\end{aligned}
$$

In a non-inferiority triple-arm trial, there are three relevant comparisons: (1) experimental treatment versus active comparator (i.e. tDCS versus escitalopram); (2) experimental treatment versus placebo; and (3) active comparator versus placebo. Authors such as Koch and Röhmel ${ }^{29}$ have considered that the third comparison is not strictly necessary if the former two were significant, but in their approach, two or three null hypotheses would have to be independently rejected (thereby decreasing the $\mathrm{P}$ value), or the trial would only be valid if all hypotheses were rejected. ${ }^{30}$ Therefore, we use here an alternative model presented by Pigeot et al. ${ }^{31}$ and already used in the literature, ${ }^{32}$ in which the study aim can be presented in a single $\mathrm{H}_{0}$, which simultaneously tests the non-inferiority between active treatments and their superiority against placebo, thereby decreasing the number of multiple comparisons.

Our secondary aims are to explore the clinical improvement in terms of response status (more than 50\% of improvement from baseline to endpoint) and remission status (HDRS-17 $\leq 7$ at endpoint). We will also explore improvement in depression using the MADRS and the BDI. Finally, we will also investigate whether any early improvement (week 3) was observed between the groups.

We also aim to identify several predictors and mediators of tDCS response, as described below.

\section{Participants}

We are recruiting patients of both genders, aged 18 to 75 years who have been diagnosed with major depressive disorder during an acute depressive episode, in accordance with the DSM-5 criteria (Diagnostic and Statistical Manual of Mental Disorders, $5^{\text {th }}$ edition).

The eligibility criteria include the presence of a depressive episode of at least moderate intensity (corresponding to a score $\geq 17$ on the 17-item Hamilton Depression Rating Scale, HDRS-17), ability to read and understand Portuguese, at least eight years of schooling and availability to adhere to the study protocol. The exclusion criteria are: 1) other neuropsychiatric conditions, such as bipolar disorder, schizophrenia, substance dependence, dementia, traumatic brain injury, epilepsy and so forth (although participants with anxiety disorders can be included if the primary diagnosis is MDD); 2) high suicide risk (i.e. score $>2$ in the Hamilton suicide question); 3) pregnancy; 4) specific contraindications against tDCS, such as electronic or metal implants in the cephalic segment; 5) specific contraindications against escitalopram; 6) severe/life-threatening clinical conditions; or 7) previous participation in other tDCS trials.

Participants will have to be either drug-naïve or drug-free regarding the use of antidepressant drugs (we considered an "antidepressant drug" to be any medication approved for treating MDD by the United States Food and Drug Administration as of the time of onset of the trial). A minimum period of 3 weeks (5 weeks for fluoxetine) will be set aside for drug washout. Benzodiazepine drugs will be allowed, although only at low doses (less than $20 \mathrm{mg} /$ day of diazepam or equivalent). Also, since escitalopram is our active comparator, patients using (or who have used) escitalopram in the current depressive episode will not be included, since they would be escitalopram-resistant.

The adherence strategies for minimizing dropouts include: reimbursement of transportation costs; flexibility in the study schedule; allowance of up to four missed visits during the acute 
study phase and up to two missed visits during the weekly tDCS phase; reminders for patients regarding their appointments; and offering active $\mathrm{tDCS}$ after the endpoint to non-responder patients who received sham $\mathrm{tDCS}$.

\section{Interventions}

We are using Soterix Medical tDCS devices specially customized for our study (Soterix Medical, New York, NY, USA, Model $1 \mathrm{x} 1 \mathrm{tDCS}-\mathrm{CT})$. For tDCS, the anode is placed over the left and the cathode over the right dorsolateral prefrontal cortex. The electrode positioning is based on the "Omni-Lateral-Electrode" (OLE) system, which is a simple, reproducible, and practical method for positioning the electrodes using a headband (Figures $2 \mathrm{~A}$ and $2 \mathrm{~B}$ ). Moreover, the OLE method optimizes electric current densities across the DLPFC, in comparison with other nonneuronavigated methods for tDCS electrode placement, such as the EEG international 10-20 system and the 5-5 cm rule (Seibt, Brunoni, Huang and Bikson, under review), taking into consideration inter-subject variability in head anatomy (Figure 2C).

The stimulation parameters are: current intensity of $2 \mathrm{~mA}$, electrode size of $25 \mathrm{~cm}^{2}$; session duration of 30 minutes (excluding the fade-in and fade-out periods of 15 seconds); and total number of 22 sessions, with 15 sessions applied consecutively once a day (except for weekends), and after that, seven more sessions applied once per week until the study endpoint at week 10. For operational reasons, these sessions are held on either Tuesdays or Thursdays, according to the patient's preference and always respecting the one-week interval.

Sham tDCS is delivered using the same procedure as active tDCS, but using a period of only 30 seconds of active stimulation at $2 \mathrm{~mA}$ (or an overall active period of 60 seconds, taking into account the 15 seconds for both the fade-in and fade-out periods), with the stimulator remaining active but not generating current for 30 minutes, for the purpose of double blinding. This method, adapted from Gandiga et al., ${ }^{33}$ has been used in several tDCS protocols ${ }^{34}$ and had the same blinding efficacy as the placebo pill in SELECT-TDCS. ${ }^{35}$ We are using fully automated devices that perform active or sham tDCS according to a randomized stimulation code.

The pharmacological intervention starts simultaneously with tDCS and consists of $10 \mathrm{mg}$ pills of escitalopram oxalate or placebo. Patients receive $10 \mathrm{mg} /$ day of escitalopram/placebo for the first 3 weeks and $20 \mathrm{mg} /$ day for the remaining 7 weeks. Escitalopram was chosen because it is an effective antidepressant treatment with few adverse effects ${ }^{36}$ and its full dose $(20 \mathrm{mg} /$ day $)$ is relatively easier to achieve than those of other antidepressant drugs. Thus, maximum dose-up titration can be done in the beginning of the trial. Therefore, we are able to compare tDCS against a full dose of an effective antidepressant without compromising blinding due to adverse effects.

The escitalopram pills are from Libbs (São Paulo, Brazil), a Brazilian pharmaceutical drug company that produces Reconter (escitalopram oxalate), which is a generic drug product comparable to the brand reference Lexapro (Lundbeck Brasil Ltda., Rio de Janeiro, Brazil). In Brazil, all generic drug products have their bioequivalence tested and certified by the Brazilian Health Surveillance Agency (ANVISA). We also independently assessed whether Reconter would be comparable to Lexapro in terms of dosage, strength and quality by testing both drugs in the Clinical Analysis Laboratory of the School of Pharmaceutical Sciences of the University of São Paulo. They achieved the same performance regarding their physical-chemical properties. The School of Pharmaceutical Sciences also produces the placebo pills, which are identical to the Reconter pills in terms of shape, color,
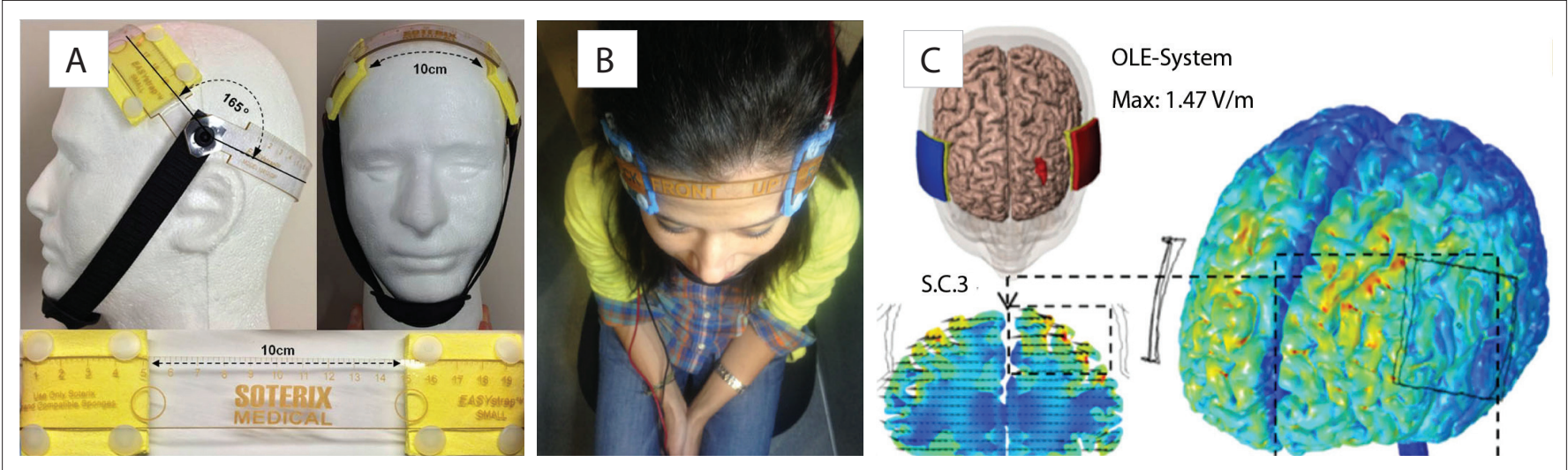

Figure 2. Electrode positioning in the ELECT-TDCS trial. (A) and (B) The figure shows the placement procedure for the Omni-LateralElectrode (OLE) system, which is placed as follows: 1) Select EasyStrap size (small, medium or large); 2) Place the midpoint of the occipital strap over inion $\left(\mathrm{I}_{\mathrm{z}}\right)$; 3) Position the hinges that link occipital-, electrode- and chin strap over the most dorsal point on the ear; 4) Adjust the angle between the occipital and electrode straps to $165^{\circ}$ and the distance across the scalp between the dorsal electrode edges to 10 $\mathrm{cm}$. Adapted from Seibt, Brunoni, Huang and Bikson (under review). (C) Brain current flow produced in one tDCS session in ELECT-TDCS. 
weight and taste. Escitalopram and placebo pills are put in identical opaque bottles, identified solely by a number corresponding to the patient's code. Adherence to the drug intervention will be verified by means of a pill count at the end of the study.

\section{Procedures}

Participants are randomized in accordance with a computergenerated list at www.randomization.com. For allocation, we use opaque sealed envelopes containing the code corresponding to the group assigned for each participant. This code is entered into the tDCS device that automatically delivers either active or sham stimulation.

Diagnoses are made by certified psychiatrists or clinical psychologists and are confirmed through the Mini-International Neuropsychiatric Interview (M.I.N.I.). The data collected include diagnosis subtype (melancholic, atypical or depressive with mixed features), duration of illness and number of failed antidepressant treatments during the current episode. The Hamilton Depression Rating Scale (HDRS-17), the Montgomery-Asberg Depression Rating Scale (MADRS), the Positive and Negative Affect Scale (PANAS) and the STAI (State-Trait Anxiety Inventory) are applied at different time points (Table 1).

To assess adverse effects, we use the Systematic Assessment for Treatment of Emergent Effects (SAFTEE) ${ }^{37}$ and the tDCS adverse events questionnaire. ${ }^{10}$ We apply the Young Mania Rating Scale (YMRS) to assess hypomanic symptoms, which have already been described after tDCS antidepressant treatment. ${ }^{38-41}$ Also, we include neuropsychological assessments in our study, primarily to verify that tDCS will not be associated with cognitive impairment, using the Montreal Cognitive Assessment (MOCA), the Wechsler Adult Intelligence Scale-III digit span (forward and backward) and digit symbol coding subtests, the Verbal Fluency
Test (FAS and animal/fruit categories) and the Trail Making test. The Temperament and Character Inventory (TCI) is being collected at baseline and endpoint to assess the personality traits associated with and modified by the antidepressant treatment. We also assess blinding performance by asking patients to guess their own treatment allocation group at week 3 and at the endpoint, and ask interviewers to make the same guesses regarding these patients' treatment.

Furthermore, we are investigating several biomarkers, including:

a) Heart rate variability (HRV), which becomes lower in cases of depression ${ }^{42}$ and possibly reflects the disrupted sympathovagal balance observed in MDD. However, recent evidence suggests that this alteration is primarily driven by the direct effects of antidepressant drug treatment. ${ }^{43}$ In our SELECT-TDCS trial, we did not observe that either tDCS or sertraline changed HRV levels, which were lower than in matched healthy controls. ${ }^{44}$ In ELECT-TDCS, we assess HRV values over a larger timeframe (of 10 weeks) and in a larger sample size.

b) Genetic polymorphisms: (i) The serotonin transporter gene (5HTTLPR, SLC6A4) is related to antidepressant response. ${ }^{45,46}$ Two out of three rTMS studies also found that it was associated with antidepressant response. ${ }^{47-49}$ In SELECT-TDCS we also found that it was associated with a larger active-sham difference. ${ }^{50}$ (ii) The $5 \mathrm{HT}_{2 \mathrm{~A}}$ receptor gene (rs6311 and rs6313) was associated with a larger active-sham difference ${ }^{51}$ and antidepressant response. ${ }^{52}$ Although a meta-analysis was inconclusive regarding its association with antidepressant response, ${ }^{46}$ it was identified as associated with citalopram antidepressant response in the $\mathrm{STAR}^{\star} \mathrm{D}$ trial. ${ }^{53}$ (iii) $\mathrm{TPH} 1$ polymorphism was associated with citalopram antidepressant response in

Table 1. Study schedule for the ELECT-TDCS

\begin{tabular}{|c|c|c|c|c|c|c|c|c|c|c|c|c|}
\hline & \multirow{2}{*}{ Triage } & & \multicolumn{10}{|c|}{ Week } \\
\hline & & 0 & 1 & 2 & 3 & 4 & 5 & 6 & 7 & 8 & 9 & 10 \\
\hline Transcranial direct current stimulation (active/sham) & & & D & D & D & W & W & W & W & W & W & W \\
\hline Escitalopram (verum/placebo) & & & D & D & D & $\mathrm{D}$ & $\mathrm{D}$ & $\mathrm{D}$ & $\mathrm{D}$ & $\mathrm{D}$ & $\mathrm{D}$ & $\mathrm{D}$ \\
\hline Triage interview & $X$ & & & & & & & & & & & \\
\hline Assessment of eligibility & $x$ & & & & & & & & & & & \\
\hline Antidepressant drug wash-out & $x$ & & & & & & & & & & & \\
\hline Genetic polymorphism collection & $x$ & & & & & & & & & & & \\
\hline Neuroimaging scans & & $X$ & & & & & & & & & & $X$ \\
\hline Clinical interviews & & $\mathrm{X}$ & $X$ & $X$ & $X$ & & & $X$ & & $X$ & & $\mathrm{X}$ \\
\hline Adverse effects & & & & & $\mathrm{X}$ & & & & & & & $X$ \\
\hline Neuropsychological evaluation & & $x$ & & & & & & & & & & $x$ \\
\hline Motor cortical excitability & & $X$ & & & $x$ & & & & & & & $X$ \\
\hline Blood collection for serum biomarkers & & $X$ & & & $X$ & & & & & & & $X$ \\
\hline Heart rate variability & & $x$ & & & $X$ & & & & & & & $X$ \\
\hline Temperament and character inventory & & $\mathrm{X}$ & & & & & & & & & & $x$ \\
\hline
\end{tabular}

"D" and " $W$ " represent interventions performed daily and weekly, respectively. 
the $\mathrm{STAR}^{\star} \mathrm{D}$ trial. The A allele was associated with lower synthesis of serotonin ${ }^{54}$ and a recent meta-analysis ${ }^{46}$ showed that this allele was associated with worse antidepressant response. (iv) Brain-derived neurotrophic factor (BDNF) polymorphism (rs6265) is a neurotrophin associated with synaptic plasticity. ${ }^{55,56}$ Two meta-analyses showed that Met/Met and Val/Met were associated with antidepressant response, ${ }^{46,57}$ while one rTMS study found that Val/Val determined a better antidepressant response. ${ }^{58}$ In SELECT-TDCS, this polymorphism was not associated with antidepressant response. ${ }^{50}$

c) Motor cortical excitability for tDCS has been used since the reappraisal of tDCS by Nitsche and Paulus in 2000. ${ }^{11}$ Currently, one important line of tDCS research involves measurement of motor cortex excitability after use of tDCS in combination with psychoactive drugs. ${ }^{59}$ This procedure allows indirect measurement of the GABAergic activity (ICI and CSP) and glutamatergic activity (ICF) of the motor system. ${ }^{60}$ These neurotransmitters are involved in the pathophysiology of depression. ${ }^{61}$ Recent studies observed that in relation to healthy subjects, the ICI, ICF and CSP indices are altered in depressed subjects, ${ }^{62-64}$ although their role as predictors of antidepressant response was not sufficiently investigated.

d) Brain imaging by means of MRI will be collected in approximately half of the sample (120 patients) at baseline and endpoint, and this imaging will include voxel-based morphometry (VBM), diffusion tensor imaging (DTI) and resting-sate functional MRI. Regarding VBM, one consistent finding observed in depressed patients is that the volume of the grey matter in the prefrontal and anterior cingulate cortex bilaterally is lower, ${ }^{65-67}$ and that there is an increase in gray matter in the left DLPFC after successful antidepressant treatment. ${ }^{68}$ These brain areas will be explored as predictors of tDCS response. On DTI, the fractional anisotropy (FA) of the prefrontal cortex (bilaterally), right temporal lobe and right fusiform gyrus were found to be lower in MDD patients. ${ }^{69}$ Studies on repetitive TMS found an increase in FA in the left frontal-medial gyrus after successful antidepressant treatment, ${ }^{70,71}$ and these regions are explored in our study. For resting-state fMRI, we explore changes in the default-mode network (DMN) and anticorrelated network (AC) after tDCS treatment. Previous studies observed that depressed patients present greater DMN activity and lower AC activity ${ }^{72}$ and that antidepressant treatment can change the brain activity of these regions. ${ }^{73}$

e) BDNF levels in blood are lower in depressed subjects than in healthy subjects and increase after successful pharmacological treatment. ${ }^{74,75}$ Nonetheless, recent meta-analyses showed that ECT increases BDNF levels in blood, but not rTMS or
tDCS. $^{76,77}$ The possible explanations for this are the low number of rTMS/tDCS studies that used non-optimal treatment protocols and the lower period of observation between measurements. In ELECT-TDCS, BDNF levels in blood are assessed at baseline, week 3 and the endpoint.

\section{Design and sample size determination}

ELECT-TDCS uses a non-inferiority, triple-arm, placebo-controlled design, with 3:3:2 permuted block randomization in which participants are respectively assigned to escitalopram, tDCS or placebo. Our aim is to prove that tDCS is non-inferior (i.e. that it has similar or superior efficacy) to escitalopram. The placebo group is used for the following purposes. (1) To ensure assay sensitivity. The placebo ascertains that a similar result between escitalopram and tDCS occurred not due to a false-positive finding caused by methodological issues such as insufficient sample size, sample bias, poor blinding, etc., but in fact due to true equivalence between treatments. (2) To allow direct (superiority) comparisons between the pharmacological treatment and placebo, which is critical, since up to $50 \%$ of antidepressant drug trials fail to detect superiority between the active treatment and placebo. ${ }^{78}$ (3) To allow direct comparisons between the experimental treatment and placebo, given that evidence of tDCS efficacy is still being established. (4) To avoid setting up an arbitrary efficacy margin, which would be a problematic approach because the active comparator in the trial will not necessarily have the same efficacy as previous findings in the literature. ${ }^{79}$ For these reasons, the non-inferiority design with a placebo group is considered to be the gold standard for this type of trial according to the US Food and Drug Administration (FDA) and the European Medical Agency (EMA). ${ }^{80}$

The $M_{2}$ margin (relative efficacy of the experimental treatment to the active comparator) is based on a fraction retention factor $(f)$ of the $M_{1}$, given that the relative efficacy of the experimental treatment to the active comparator could only be larger than the efficacy with placebo if the active comparator is worse than placebo. The fraction retention varies according to the condition investigated: for instance, in studies focusing on vaccines and oncology, the $f$ value should be close to 1; whereas for chronic and functional disorders, this value ranges between one-half and one-third. ${ }^{31,79}$ There is no consensus regarding the $f$ value in non-inferiority trials for MDD, although Nutt et al. ${ }^{81}$ proposed that for generalized anxiety disorder, the value should be $50 \%$. In this trial, we adopted the $f$ value of $50 \%$, based on Nutt et al. ${ }^{81}$ and considering that MDD is comparable to generalized anxiety disorder with regard to methodological aspects (large placebo response, short duration of clinical trials, similar pharmacological treatments, comparable scales etc.). 
Therefore, in our trial, $\mathrm{M}_{2} / \mathrm{M}_{1}=0.5$, although $\mathrm{M}_{1}$ is based on the difference between the active comparator and placebo and it will be known only when the study has been finished. Although this approach indexes the relative efficacy of tDCS versus escitalopram according to the placebo response of the study, it is theoretically possible that the escitalopram-placebo difference is too large, thus producing a large $\mathrm{M}_{2}$ value that could favor a finding that comparison between escitalopram and tDCS does not show any significant difference, even if escitalopram clinically outperforms tDCS. To avoid this issue, we will only consider that tDCS was non-inferior to escitalopram if the mean difference between escitalopram and tDCS is less than 3 points on the HDRS-17. The threshold of 3 points was chosen considering the National Institute for Health and Clinical Excellence (NICE) guidelines, which establish that this is a clinically meaningful difference in efficacy. ${ }^{82}$

To determine the sample size, we first needed to establish the randomization proportions between the groups. Pigeot et al. ${ }^{31}$ recommended a 1:1: $\mathrm{k}_{\mathrm{p}}$ ratio, where $\mathrm{k}_{\mathrm{p}}$ is determined according to the fraction retention factor, as shown below:

$\mathrm{k}_{\text {placebo }}=[(1-f) \sqrt{ } 2+2 f] /\left(1+f^{2}\right)$

According to this formula, for $f=0.5, \mathrm{k}_{\mathrm{p}}$ is 0.69 . Therefore the optimal proportion for allocation between the groups is 1:1:0.69, or approximately 3:3:2, as used in ELECT-TDCS.

We estimated our sample size in accordance with the suggestion of Pigeot et al. ${ }^{31}$ for non-inferiority, triple-arm trials:

$N_{\text {arm }} \geq\left(\mathrm{t}_{1-\alpha, 3 \mathrm{n}-3}+\mathrm{t}_{1-\beta, 3 \mathrm{n}-3}\right)^{2 *}\left(1+f^{2}+(1-f)^{2}\right)^{*}\left[\left(\sigma / \mu_{\mathrm{DRUG}}-\mu_{\mathrm{PLACEBO}}\right) /(\mathrm{r}-f)\right]^{2 *}$

This formula shows that the sample size is a product of three factors (in parentheses). The factor in the first parentheses take into account the values of $\alpha$ and $\beta$, which were, respectively 0.025 (one-tailed) and 0.2. The factor in the second parentheses is the $f$ value (the higher the $f$ value is, the larger the necessary sample size will be), which was determined as 0.5 for our study. The factor in the last parentheses involves the standard deviation and effect sizes of the interventions. We took the value of the standard deviation to be a function of the difference in efficacy between the active comparator and the placebo: ${ }^{31}$

$\sigma=\varepsilon\left(\mu_{\mathrm{DRUG}}-\mu_{\mathrm{PLACEBO}}\right)$

The $\varepsilon$ value indicates that the standard deviation is associated with the mean difference values observed in the study. It ranges from 0.2 to 2 , and values $>1$ are considered conservative. In the

\footnotetext{
*Where $\sigma$ is the standard deviation of the study and $r=\left(\mu_{\text {tDCS }}-\mu_{\text {PLACEBO }}\right) /$

$\left(\mu_{\text {DRUG }}-\mu_{\text {PLACEBO }}\right)$. If $\mu_{\text {tDCS }}=\mu_{\text {DRUG }}$ then $r=1$.
}

present study, we took the $\varepsilon$ value to be 1.5 , i.e. the standard deviation would be 1.5 times the value of the difference in efficacy between the active intervention and the placebo, which is compatible with depression studies in which the variance is usually high. ${ }^{\dagger}$

Therefore, considering an attrition rate of $13 \%$ (similar to SELECT-TDCS), $\beta=0.2, \alpha_{\text {one-tailed }}=0.025, f=0.5$ and $\varepsilon=1.5$, a total sample of 240 patients will be necessary in order to reject our null hypothesis. For 3:3:2 randomization, this means that in the end, 90, 90 and 60 patients will be respectively allocated to the tDCS, escitalopram and placebo groups.

\section{Statistical analysis}

In accordance with the recommendations of Pigeot et al. ${ }^{31}$ and Rothmann et al. ${ }^{79}$ we use a modified $t$ test to address our primary study hypothesis (i.e. that $\mathrm{tDCS}$ is non-inferior to escitalopram). A $t$ test involves obtaining a $T$ value, which should be higher than (in a one-tailed test, as in our study) or different to (in a twotailed test) a critical $t$ value. The $\mathrm{T}$ value is obtained by dividing the difference in the means by the mean error (obtained according to the variance and sample size). For this analysis, the T value will be obtained as follows:

$\mathrm{T}=\left(\mu_{\mathrm{tDCS}}-0.5 \mu_{\text {PLACEBO }}-0.5 \mu_{\text {DRUG }}\right) / \sigma \sqrt{ }\left(1 / \mathrm{n}_{\text {TDCS }}+0.25 / \mathrm{n}_{\text {DRUG }}+\right.$ $\left.0.25 / \mathrm{n}_{\text {PLACEBO }}\right)$ or

$\mathrm{T}=7.44\left(\mu_{\mathrm{tDCS}}-0.5 \mu_{\text {PLACEBO }}-0.5 \mu_{\mathrm{DRUG}}\right) / \sigma$ (considering that the $\mathrm{n}$ values are known)

This $\mathrm{T}$ value should be higher than the critical $t$, which for a one tailed $\alpha$ of 0.025 and 237 (N-3) degrees of freedom would be 1.97.

We will also perform exploratory analyses to identify whether a significant difference between the groups is observed over time. Therefore, we will perform mixed-model analyses of variance (ANOVAs) with one independent, within-subject variable (time, with three levels: baseline, week 3 and endpoint) and one independent, between-subject variable (group, with three levels). The dependent variables will be the scores from HDRS, MADRS or BDI. To determine whether a statistical significance is observed at a two-tailed $\mathrm{p}$ value of 0.05 or less, post-hoc analyses will be performed to analyze the main and interaction effects of our interventions. Exploratory analyses will also be performed to identify the influence of clinical, demographic and biological variables on the outcome. In addition, logistic regressions will be performed using response or remission as dependent variables and the group as an independent variable. Adverse effects will be assessed by counting the number

${ }^{\dagger}$ For comparative purposes, these values were as follows in SELECT-TDCS: $\mu_{\mathrm{tDCS}}=7.7, \mu_{\text {PLACEBO }}=3.6$ and $\sigma=6.3$ for the HDRS-17. Therefore, $\varepsilon=6.3 /(7.7-3.6)$, or 1.53 (considering that $\mu_{\mathrm{tDCS}}=\mu_{\mathrm{DRUG}}$ ). 
of events in each group at each evaluation time. The chi-square test will be used to compare the frequency of adverse effects between the treatment groups.

For non-inferiority trials, it is unclear which approach (intention-to-treat, ITT; or per protocol, PP) should be used. ITT imputes data from study drop-outs and, although conservative for superiority trials, it can favor the hypothesis of lack of difference between groups in a non-inferiority design and, therefore, benefit (instead of penalizing) a non-inferiority design with methodological flaws. Nonetheless, neither the $\mathrm{FDA}^{83}$ nor the $\mathrm{EMA}^{84}$ clearly recommend the optimal approach and they suggest that both ITT and PP should be used. Mulla et al. ${ }^{85}$ also suggested that ITT should be used because it has the additional benefit of being more robust in relation to bias, compared with PP analysis. Therefore, missing data will be handled using an ITT approach and the findings will be confirmed through PP analysis. Finally, we will perform additional analyses according to the patients' adherence to the study, using the categories of "fully adherent sample" (patients who missed or rescheduled two visits or fewer) and "completer sample" (patients who completed the study in accordance with the protocol). This approach was also used by George et al. ${ }^{6}$

\section{DISCUSSION}

ELECT-TDCS will be the largest trial to date assessing the efficacy of tDCS and the first designed to specifically compare the efficacy of tDCS with a full dose of escitalopram. Demonstrating that tDCS presents efficacy similar to that of a pharmacological treatment is important, because antidepressant drugs present several relative and some absolute contraindications against use. Therefore, tDCS could increase the therapeutic arsenal for use among depressed patients who cannot or are not willing to use antidepressant drugs, e.g. pregnant women, ${ }^{86} \mathrm{HIV}$ patients ${ }^{87}$ and patients presenting clinical conditions in which the pharmacokinetic interactions are problematic. $^{88}$

Our study is also interesting from a cost-efficacy perspective, since tDCS is an affordable, portable and ease-to-use therapy. The cost of antidepressant therapy consisting of tDCS might, in fact, be comparable to that of antidepressant drugs, if it is taken into consideration that one tDCS device can be used to perform several applications per day and that one operator can deliver tDCS to two to three patients simultaneously. Finally, tDCS is a technique with few adverse effects. ${ }^{10}$ There are at present no reports of seizures or other severe life-threatening events. The most serious adverse effect reported hitherto is skin burn at the application site, and this has been an uncommon finding. ${ }^{89}$

Another key aspect of ELECT-TDCS is that several biomarkers will be assessed in a multimodal approach. tDCS does not seem to induce peripheral effects in cases of depression, such as changes to blood neurotrophic factors. ${ }^{77,90,91}$ However, it increases central neuroplasticity as indexed by paired associative stimulation, ${ }^{92}$ modulates cortical activity as indexed by electroencephalography ${ }^{93}$ and improves working memory and affective processing. ${ }^{94-96}$ Finally, since the same analyses will be performed in the escitalopram group, we will also explore whether the biological predictors and mediators are different between tDCS and escitalopram responders.

Our study protocol was designed by drawing on our experience from our earlier SELECT-TDCS trial and other advances observed in the field. The choice of using escitalopram at a dose of $20 \mathrm{mg} /$ day represents an advance over the $50 \mathrm{mg} /$ day dose of sertraline used in SELECT-TDCS. The decision to increase the total number of sessions from 12 to 22 was based on findings from a meta-analysis ${ }^{24}$ that suggested that a higher tDCS "dose" would be associated with a larger improvement in depression. Other changes in relation to SELECT-TDCS include: a longer wash-out period to ensure that the subjects were truly "antidepressant drug-free"; an increase in the maximum age of participants in order to improve recruitment; use of automated tDCS devices for sham stimulation, so as to improve blinding; a longer study duration to address the effects of tDCS over a longer time frame; and use of HDRS-17 (instead of MADRS) as the primary outcome measurement, given that in SELECT-TDCS, HDRS17 presented lower variance and greater psychometric validity (Cronbach's alpha and intraclass correlation) than MADRS, and was also more sensitive towards detecting between-group differences (data not published).

Nonetheless, most aspects of the SELECT-TDCS trial were maintained, such as recruitment of unipolar depressed patients with different degrees of refractoriness in an acute episode of at least moderate severity; enrollment of antidepressant-free patients; inclusion of patients using benzodiazepine drugs, since we considered that exclusion of these patients could harm recruitment and, in fact, be ineffective given that participants can retain information regarding use of these drugs; bilateral (leftright) DLPFC stimulation; and comparison with a placebo arm, which is crucial, given that as the efficacy of active versus sham tDCS is still under investigation.

\section{Limitations}

ELECT-TDCS also presents some limitations. First, tDCS is being compared with escitalopram and therefore the results will not be fully generalizable to other antidepressant drugs. Second, we are not testing other tDCS montages and parameters that have also improved depressive symptoms. Third, we are not testing the combination of tDCS with pharmacotherapy or cognitive-behavioral therapy. Finally, our multimodal analyses are exploratory. 


\section{CONCLUSIONS}

The ELECT-TDCS trial is a 10 -week, phase-III, non-inferiority, triple-arm, placebo-controlled study that is investigating whether tDCS is non-inferior (i.e. whether it has similar or superior efficacy) to $20 \mathrm{mg}$ /day of escitalopram. The results from our trial have the potential to introduce a novel technique into the therapeutic arsenal for depression treatment, particularly in primary care contexts or among patients who cannot tolerate or are not willing to use antidepressant drugs. Our multimodal investigation of biomarkers will also contribute towards understanding the antidepressant mechanisms of the effects of $\mathrm{tDCS}$.

\section{REFERENCES}

1. Bromet E, Andrade LH, Hwang I, et al. Cross-national epidemiology of DSM-IV major depressive episode. BMC Med. 2011;9:90.

2. Murray CJ, Lopez AD. Alternative projections of mortality and disability by cause 1990-2020: Global Burden of Disease Study. Lancet. 1997;349(9064):1498-504.

3. Rush AJ, Trivedi MH, Wisniewski SR, et al. Acute and longerterm outcomes in depressed outpatients requiring one or several treatment steps: a STAR*D report. Am J Psychiatry. 2006; 163(11):1905-17.

4. Anderson IM, Ferrier IN, Baldwin RC, et al. Evidence-based guidelines for treating depressive disorders with antidepressants: a revision of the 2000 British Association for Psychopharmacology guidelines. J Psychopharmacol. 2008;22(4):343-96.

5. Rosa MA, Lisanby SH. Somatic treatments for mood disorders. Neuropsychopharmacology. 2012;37(1):102-16.

6. George MS, Lisanby SH, Avery D, et al. Daily left prefrontal transcranial magnetic stimulation therapy for major depressive disorder: a shamcontrolled randomized trial. Arch Gen Psychiatry. 2010;67(5):507-16.

7. Schutter DJ. Antidepressant efficacy of high-frequency transcranial magnetic stimulation over the left dorsolateral prefrontal cortex in double-blind sham-controlled designs: a meta-analysis. Psychol Med. 2009;39(1):65-75.

8. Priori A, Hallett $M$, Rothwell JC. Repetitive transcranial magnetic stimulation or transcranial direct current stimulation? Brain Stimul. 2009;2(4):241-5.

9. Brunoni AR, Nitsche MA, Bolognini N, et al. Clinical research with transcranial direct current stimulation (tDCS): challenges and future directions. Brain Stimul. 2012;5(3):175-95.

10. Brunoni AR, Amadera J, Berbel B, et al. A systematic review on reporting and assessment of adverse effects associated with transcranial direct current stimulation. Int J Neuropsychopharmacol. 2011;14(8):1133-45.

11. Nitsche MA, Paulus W. Excitability changes induced in the human motor cortex by weak transcranial direct current stimulation. J Physiol. 2000;527 Pt 3:633-9.
12. Purpura DP, McMurtry JG. Intracellular activities and evoked potential changes during polarization of motor cortex. J Neurophysiol. 1965;28:166-85.

13. Radman T, Ramos RL, Brumberg JC, Bikson M. Role of cortical cell type and morphology in subthreshold and suprathreshold uniform electric field stimulation in vitro. Brain Stimul. 2009;2(4): 215-28, 228. e1-3.

14. Batsikadze G, Moliadze V, Paulus W, Kuo MF, Nitsche MA. Partially non-linear stimulation intensity-dependent effects of direct current stimulation on motor cortex excitability in humans. J Physiol. 2013;591(Pt 7):1987-2000.

15. Rahman A, Reato D, Arlotti M, et al. Cellular effects of acute direct current stimulation: somatic and synaptic terminal effects. J Physiol. 2013;591(Pt 10):2563-78.

16. Mayberg HS, Brannan SK, Tekell JL, et al. Regional metabolic effects of fluoxetine in major depression: serial changes and relationship to clinical response. Biol Psychiatry. 2000;48(8):830-43.

17. Moffa AH, Valiengo L, Shiozawa P, Brunoni AR. Novas neuroterapêuticas em psiquiatria: racional e uso da estimulação transcraniana por corrente contínua no transtorno depressivo maior [Novel neurotherapeutics in psychiatry: use and rationale of transcranial direct current stimulation in major depressive disorder]. Rev Psiquiatr Clín. (Sao Paulo). 2014;41 (1):15-20.

18. Ferrucci R, Bortolomasi M, Brunoni A, et al. Comparative benefits of transcranial direct current stimulation (tDCS) treatment in patients with mild/moderate vs. severe depression. Clinical Neuropsychiatry. 2009;6(6):246-51. Available from: http://www.clinicalneuropsychiatry. org/pdf/03\%20ferrucci.pdf. Accessed in 2015 (Jan 7).

19. Schutter DJ. Quantitative review of the efficacy of slow-frequency magnetic brain stimulation in major depressive disorder. Psychol Med. 2010;40(11):1789-95.

20. Brunoni AR, Ferrucci R, Fregni F, Boggio PS, Priori A. Transcranial direct current stimulation for the treatment of major depressive disorder: a summary of preclinical, clinical and translational findings. Prog Neuropsychopharmacol Biol Psychiatry. 2012;39(1):9-16.

21. Fregni F, Boggio PS, Nitsche MA, et al. Treatment of major depression with transcranial direct current stimulation. Bipolar Disord. 2006;8(2):203-4.

22. Loo CK, Alonzo A, Martin D, et al. Transcranial direct current stimulation for depression: 3-week, randomised, sham-controlled trial. Br J Psychiatry. 2012;200(1):52-9.

23. Brunoni $A R$, Valiengo $L$, Baccaro $A$, et al. The sertraline vs. electrical current therapy for treating depression clinical study: results from a factorial, randomized, controlled trial. JAMA Psychiatry. 2013;70(4):383-91.

24. Shiozawa P, Fregni F, Benseens IM, et al. Transcranial direct current stimulation for major depression: an updated systematic review and meta-analysis. Int J Neuropsychopharmacol. 2014;17(9):1443-52. 
25. Loo CK, Sachdev P, Martin D, et al. A double-blind, sham-controlled trial of transcranial direct current stimulation for the treatment of depression. Int J Neuropsychopharmacol. 2010;13(1):61-9.

26. Palm U, Schiller C, Fintescu Z, et al. Transcranial direct current stimulation in treatment resistant depression: a randomized doubleblind, placebo-controlled study. Brain Stimul. 2012;5(3):242-51.

27. Blumberger DM, Tran LC, Fitzgerald PB, Hoy KE, Daskalakis ZJ. A randomized double-blind sham-controlled study of transcranial direct current stimulation for treatment-resistant major depression. Front Psychiatry. 2012;3:74.

28. Brunoni $A R$, Valiengo $L$, Baccaro $A$, et al. Sertraline vs. Electrical Current Therapy for Treating Depression Clinical Trial--SELECT TDCS: design, rationale and objectives. Contemp Clin Trials. 2011;32(1):90-8.

29. Koch A, Roch AJ. Hypothesis testing in the "gold standard" design for proving the efficacy of an experimental treatment relative to placebo and a reference. J Biopharm Stat. 2004;14(2):315-25.

30. Gamalo MA, Muthukumarana S, Ghosh P, Tiwari RC. A generalized p-value approach for assessing noninferiority in a three-arm trial. Stat Methods Med Res. 2013;22(3):261-77.

31. Pigeot I, Schafer J, Rohmel J, Hauschke D. Assessing non-inferiority of a new treatment in a three-arm clinical trial including a placebo. Stat Med. 2003;22(6):883-99.

32. Binkley $N$, Bolognese $M$, Sidorowicz-Bialynicka A, et al. A phase 3 trial of the efficacy and safety of oral recombinant calcitonin: the Oral Calcitonin in Postmenopausal Osteoporosis (ORACAL) trial. J Bone Miner Res. 2012;27(8):1821-9.

33. Gandiga PC, Hummel FC, Cohen LG. Transcranial DC stimulation (tDCS): a tool for double-blind sham-controlled clinical studies in brain stimulation. Clin Neurophysiol. 2006;117(4):845-50.

34. Nitsche MA, Cohen LG, Wassermann EM, et al. Transcranial direct current stimulation: State of the art 2008. Brain Stimul. 2008;1 (3):206-23.

35. Brunoni $A R$, Schestatsky $P$, Lotufo $P A$, Benseñor IM, Fregni $F$. Comparison of blinding effectiveness between sham tDCS and placebo sertraline in a 6-week major depression randomized clinical trial. Clin Neurophysiol. 2014;125(2):298-305.

36. Cipriani A, Furukawa TA, Salanti G, et al. Comparative efficacy and acceptability of 12 new-generation antidepressants: a multipletreatments meta-analysis. Lancet. 2009;373(9665):746-58.

37. Rabkin JG, Markowitz JS, Ocepek-Welikson K, Wager SS. General versus systematic inquiry about emergent clinical events with SAFTEE: implications for clinical research. J Clin Psychopharmacol. 1992;12(1):3-10.

38. Baccaro A, Brunoni AR, Benseñor IM, Fregni F. Hypomanic episode in unipolar depression during transcranial direct current stimulation. Acta Neuropsychiatrica. 2010;22(6):316-8. Available from: http:// onlinelibrary.wiley.com/doi/10.1111/j.1601-5215.2010.00495.x/ abstract. Accessed in 2015 (Jan 7).

39. Brunoni AR, Valiengo L, Zanao T, et al. Manic psychosis after sertraline and transcranial direct-current stimulation. J Neuropsychiatry Clin Neurosci. 2011;23(3):E4-5.
40. Arul-Anandam AP, Loo C, Mitchell P. Induction of hypomanic episode with transcranial direct current stimulation. J ECT. 2010;26(1):68-9.

41. Gálvez V, Alonzo A, Martin S, et al. Hypomania induction in a patient with bipolar II disorder by transcranial direct current stimulation (tDCS). J ECT. 2011;27(3):256-8.

42. Kemp AH, Quintana DS, Gray MA. Impact of depression and antidepressant treatment on heart rate variability: a review and meta-analysis. Biol Psychiatry. 2010;67(11):1067-74.

43. Kemp AH, Brunoni AR, Santos IS, et al. Effects of depression, anxiety, comorbidity, and antidepressants on resting-state heart rate and its variability: an ELSA-Brasil cohort baseline study. Am J Psychiatry. 2014;171(2):1328-34.

44. Brunoni AR, Kemp AH, Dantas EM, et al. Heart rate variability is a trait marker of major depressive disorder: evidence from the sertraline vs. electric current therapy to treat depression clinical study. Int J Neuropsychopharmacol. 2013;16(9):1937-49.

45. Crisafulli C, Fabbri C, Porcelli S, et al. Pharmacogenetics of antidepressants. Front Pharmacol. 2011;16;2:6.

46. Kato M, Serretti A. Review and meta-analysis of antidepressant pharmacogenetic findings in major depressive disorder. Mol Psychiatry. 2010;15(5):473-500.

47. Zanardi R, Magri L, Rossini D, et al. Role of serotonergic gene polymorphisms on response to transcranial magnetic stimulation in depression. Eur Neuropsychopharmacol. 2007;17(10):651-7.

48. Bocchio-Chiavetto L, Bagnardi V, Zanardini R, et al. Serum and plasma BDNF levels in major depression: a replication study and metaanalyses. World J Biol Psychiatry. 2010;11(6):763-73.

49. Malaguti A, Rossini D, Lucca A, et al. Role of COMT, 5-HT(1A), and SERT genetic polymorphisms on antidepressant response to Transcranial Magnetic Stimulation. Depress Anxiety. 2011;28(7):568-73.

50. Brunoni AR, Kemp AH, Shiozawa P, et al. Impact of 5-HTTLPR and BDNF polymorphisms on response to sertraline versus transcranial direct current stimulation: implications for the serotonergic system. Eur Neuropsychopharmacol. 2013;23(11):1530-40.

51. Du L, Bakish D, Lapierre YD, Ravindran AV, Hrdina PD. Association of polymorphism of serotonin $2 \mathrm{~A}$ receptor gene with suicidal ideation in major depressive disorder. Am J Med Genet. 2000;96(1):56-60.

52. Van Oekelen D, Luyten WH, Leysen JE. 5-HT2A and 5-HT2C receptors and their atypical regulation properties. Life Sci. 2003;72(22):2429-49.

53. McMahon FJ, Buervenich S, Charney D, et al. Variation in the gene encoding the serotonin $2 \mathrm{~A}$ receptor is associated with outcome of antidepressant treatment. Am J Hum Genet. 2006;78(5):804-14.

54. Jonsson EG, Goldman D, Spurlock G, et al. Tryptophan hydroxylase and catechol-O-methyltransferase gene polymorphisms: relationships to monoamine metabolite concentrations in CSF of healthy volunteers. Eur Arch Psychiatry Clin Neurosci. 1997;247(6):297-302.

55. Ventimiglia $\mathrm{R}$, Mather $\mathrm{PE}$, Jones $\mathrm{BE}$, Lindsay RM. The neurotrophins BDNF, NT-3 and NT-4/5 promote survival and morphological and biochemical differentiation of striatal neurons in vitro. Eur J Neurosci. 1995;7(2):213-22. 
56. Kuipers SD, Trentani A, Den Boer JA, Ter Horst GJ. Molecular correlates of impaired prefrontal plasticity in response to chronic stress. J Neurochem. 2003;85(5):1312-23.

57. Domschke K, Lawford B, Laje G, et al. Brain-derived neurotrophic factor (BDNF) gene: no major impact on antidepressant treatment response. Int J Neuropsychopharmacol. 2010;13(1):93-101.

58. Bocchio-Chiavetto L, Miniussi C, Zanardini R, et al. 5-HTTLPR and BDNF Val66Met polymorphisms and response to rTMS treatment in drug resistant depression. Neurosci Lett. 2008;437(2):130-4.

59. Stagg CJ, Nitsche MA. Physiological basis of transcranial direct current stimulation. Neuroscientist. 2011;17(1):37-53.

60. Kobayashi M, Pascual-Leone A. Transcranial magnetic stimulation in neurology. Lancet Neurol. 2003;2(3):145-56.

61. Hasler G, Northoff G. Discovering imaging endophenotypes for major depression. Mol Psychiatry. 2011;16(6):604-19.

62. Bajbouj M, Lisanby SH, Lang UE, et al. Evidence for impaired cortical inhibition in patients with unipolar major depression. Biol Psychiatry. 2006;59(5):395-400.

63. Levinson AJ, Fitzgerald PB, Favalli G, et al. Evidence of cortical inhibitory deficits in major depressive disorder. Biol Psychiatry. 2010;67(5):458-64.

64. Croarkin PE, Nakonezny PA, Husain MM, et al. Evidence for increased glutamatergic cortical facilitation in children and adolescents with major depressive disorder. JAMA Psychiatry. 2013;70(3):291-9.

65. Lai CH. Gray matter volume in major depressive disorder: a metaanalysis of voxel-based morphometry studies. Psychiatry Res. 2013;211(1):37-46.

66. Du MY, Wu QZ, Yue Q, et al. Voxelwise meta-analysis of gray matter reduction in major depressive disorder. Prog Neuropsychopharmacol Biol Psychiatry. 2012;36(1):11-6.

67. Bora E, Fornito A, Pantelis C, Yucel M. Gray matter abnormalities in Major Depressive Disorder: a meta-analysis of voxel based morphometry studies. J Affect Disord. 2012;138(1-2):9-18.

68. Smith R, Chen K, Baxter L, Fort C, Lane RD. Antidepressant effects of sertraline associated with volume increases in dorsolateral prefrontal cortex. J Affect Disord. 2013;146(3):414-9.

69. Liao $Y$, Huang $X, W u$ Q, et al. Is depression a disconnection syndrome? Meta-analysis of diffusion tensor imaging studies in patients with MDD. J Psychiatry Neurosci. 2013;38(1):49-56.

70. Kozel FA, Johnson KA, Nahas Z, et al. Fractional anisotropy changes after several weeks of daily left high-frequency repetitive transcranial magnetic stimulation of the prefrontal cortex to treat major depression. J ECT. 2011;27(1):5-10.

71. Peng $H$, Zheng $H$, Li L, et al. High-frequency rTMS treatment increases white matter FA in the left middle frontal gyrus in young patients with treatment-resistant depression. J Affect Disord. 2012;136(3):249-57.

72. Wang L, Hermens DF, Hickie IB, Lagopoulos J. A systematic review of resting-state functional-MRI studies in major depression. J Affect Disord. 2012;142(1-3):6-12.
73. Posner J, Hellerstein DJ, Gat I, et al. Antidepressants normalize the default mode network in patients with dysthymia. JAMA Psychiatry. 2013;70(4):373-82.

74. Brunoni AR, Lopes M, Fregni F. A systematic review and metaanalysis of clinical studies on major depression and BDNF levels: implications for the role of neuroplasticity in depression. Int J Neuropsychopharmacol. 2008;11(8):1169-80.

75. Molendijk ML, Spinhoven P, Polak M, et al. Serum BDNF concentrations as peripheral manifestations of depression: evidence from a systematic review and meta-analyses on 179 associations ( $N=9484)$. Mol Psychiatry. 2014;19(7):791-800.

76. Brunoni AR, Baeken C, Machado-Vieira R, Gattaz WF, Vanderhasselt MA. BDNF blood levels after electroconvulsive therapy in patients with mood disorders: a systematic review and meta-analysis. World J Biol Psychiatry. 2014;15(5):411-8.

77. Brunoni AR, Machado-Vieira R, Zarate CA Jr., et al. BDNF plasma levels after antidepressant treatment with sertraline and transcranial direct current stimulation: results from a factorial, randomized, shamcontrolled trial. Eur Neuropsychopharmacol. 2014;24(7):1144-51.

78. Turner EH, Matthews AM, Linardatos E, Tell RA, Rosenthal R. Selective publication of antidepressant trials and its influence on apparent efficacy. N Engl J Med. 2008;358(3):252-60.

79. Rothmann MD, Wiens BL, Chan ISF. Design and analysis of noninferiority trials. Boca Raton: Taylor \& Francis Group; 2012.

80. Huitfeldt B, Hummel J, European Federation of Statisticians in the Pharmaceutical Industry (EFSPI). The draft FDA guideline on non-inferiority clinical trials: a critical review from European pharmaceutical industry statisticians. Pharm Stat. 2011;10(5):414-9.

81. Nutt D, Allgulander C, Lecrubier Y, Peters T, Wittchen U. Establishing non-inferiority in treatment trials in psychiatry: guidelines from an Expert Consensus Meeting. J Psychopharmacol. 2008;22(4):409-16.

82. Middleton $H$, Shaw I, Hull S, Feder G. NICE guidelines for the management of depression. BMJ. 2005;330(7486):267-8.

83. Guidance for Industry Non-Inferiority Clinical Trials; 2010. Available from: http://www.fda.gov/downloads/Drugs/ GuidanceComplianceRegulatorylnformation/Guidances/ UCM202140.pdf. Accessed in 2015 (Jan 7).

84. European Medicines Agency. Pre-authorisation Evaluation of Medicines for Human Use. Guideline on the choice of the noninferiority margin; 2004. Available from: http://www.emea.europa. eu/docs/en_GB/document_library/Scientific_guideline/2009/09/ WC500003636.pdf. Accessed in 2015 (Jan 7).

85. Mulla SM, Scott IA, Jackevicius CA, You JJ, Guyatt GH. How to use a noninferiority trial: users' guides to the medical literature. JAMA. 2012;308(24):2605-11.

86. Vigod S, Dennis CL, Daskalakis Z, et al. Transcranial direct current stimulation (tDCS) for treatment of major depression during pregnancy: study protocol for a pilot randomized controlled trial. Trials. 2014;15:366. 
87. Knotkova H, Rosedale M, Strauss SM, et al. Using Transcranial Direct Current Stimulation to Treat Depression in HIV-Infected Persons: The Outcomes of a Feasibility Study. Front Psychiatry. 2012;3:59.

88. Valiengo LC, Benseñor IM, Lotufo PA, Fraguas R Jr, Brunoni AR. Transcranial direct current stimulation and repetitive transcranial magnetic stimulation in consultation-liaison psychiatry. Braz J Med Biol Res. 2013;46(10):815-23.

89. Palm U, Keeser D, Schiller $C$, et al. Skin lesions after treatment with transcranial direct current stimulation (tDCS). Brain Stimul. 2008;1(4):386-7.

90. Palm U, Fintescu Z, Obermeier M, et al. Serum levels of brain-derived neurotrophic factor are unchanged after transcranial direct current stimulation in treatment-resistant depression. J Affect Disord. 2013;150(2):659-63.

91. Brunoni AR, Machado-Vieira R, Zarate CA Jr., et al. Assessment of non-BDNF neurotrophins and GDNF levels after depression treatment with sertraline and transcranial direct current stimulation in a factorial, randomized, sham-controlled trial (SELECT-TDCS): An exploratory analysis. Prog Neuropsychopharmacol Biol Psychiatry. 2015;56:91-6.

92. Player M, Taylor J, Weickert CS, et al. Increase in PAS-induced neuroplasticity after a treatment course of transcranial direct current stimulation for depression. J Affect Disord. 2014;167:140-7.

93. Powell TY, BoonstraTW, Martin DM, Loo CK, Breakspear M. Modulation of cortical activity by transcranial direct current stimulation in patients with affective disorder. PLoS One. 2014;9(6):e98503.

94. Wolkenstein L, Plewnia C. Amelioration of cognitive control in depression by transcranial direct current stimulation. Biol Psychiatry. 2013;73(7):646-51.

95. Oliveira JF, Zanao TA, Valiengo L, et al. Acute working memory improvement after tDCS in antidepressant-free patients with major depressive disorder. Neurosci Lett. 2013;537:60-4.

96. Zanao TA, Moffa AH, Shiozawa P, et al. Impact of Two or Less Missing Treatment Sessions on tDCS Clinical Efficacy: Results From a Factorial, Randomized, Controlled Trial in Major Depression. Neuromodulation. 2014;17(8):737-742.

Acknowledgements: ELECT-TDCS is funded by a FAPESP Young Researcher grant from the São Paulo State Foundation (FAPESP, grant number 20911-5). ARB is also supported by a 2013 NARSAD Young Investigator grant from the Brain \& Behavior Research Foundation (grant number 20493) and a 2013 National Council for Scientific and Technological Development grant (CNPq, grant number 470904). BSPJ is supported by a fellowship from the Brain \& Behavior Research Foundation (grant number 20493). BV and MM are supported by Scientific Initiation fellowships from FAPESP (2013/19759-7 and 2013/19566-4, respectively). AHM and BSN are supported by CNPq Master's fellowships. The authors are grateful to the following staff members who are being of invaluable help in implementing the study:
Cibele Soares, Shirley L. Santos and Amanda Campos (study nurses) and Roberta F. de Mello (study coordinator). The authors are also grateful to Geraldo Busatto, MD, PhD, and Edson Amaro Jr., MD, PhD, who are responsible for the neuroimaging aspects of the study; Paulo A. Lotufo, MD, PhD, for the administrative support; Humberto Ferraz, PhD, who is the head of the laboratory in charge of manufacturing the placebo pills; Alexandre Pereira, MD, PhD, who is responsible for the genetic analysis of the study; Ligia Maria Fedeli, BSc, and Stephany Vidal, BSc, who are responsible for storing the blood samples; and Marie-Anne Vanderhasselt, PhD, for thoughtful discussion on some aspects of the study and providing expertise in neuropsychological assessments

Authors' contributions: ARB, RF and IMB were responsible for the design and conception of the study. BSPJ, AHM, LB, BSN, LAA, BV, MM, RAF, DT and PVSB participated in data collection (recruitment, clinical interviews, motor cortical excitability and brain scans). MB and OS were responsible for the design of the sham intervention and for the new method regarding electrode positioning. All authors were critically involved in drafting parts of the manuscript and reviewing it as a whole. All authors gave final approval for the version to be published and agree to be accountable for all aspects of the ongoing trial

\section{Sources of funding: None \\ Conflict of interest: None}

\section{Submitted: November 24, 2014 \\ Last updated: December 17, 2014 \\ Accepted: December 17, 2014}

\section{Address for correspondence:}

André Russowsky Brunoni, MD, PhD

Av. Prof. Lineu Prestes, 2.565 — 3andar — Centro de Pesquisas Clínicas

Hospital Universitário (USP)

Cidade Universitária — São Paulo (SP) — Brasil

CEP 05508-000

Tel./Fax. (+55 11) 3091-9241

E-mail: brunowsky@gmail.com 